$\mathrm{DE}$

M E D I C I N A

T R O P I C A L

$\mathrm{DE}$

SÃO PAULO

JOURNAL OF THE SÃO PAULO INSTITUTE OF TROPICAL MEDICINE

${ }^{1}$ Hospital Unimed Resende, Resende, Rio de Janeiro, Brazil

${ }^{2}$ Riscobiologico.org, Rio de Janeiro, Rio de Janeiro, Brazil

*These authors contributed equally to this work

Correspondence to: João Guimarães

Ferreira

Hospital Unimed Resende, Rua Dra.

Raphaela Maria Bruno, $\mathrm{s} / \mathrm{n}^{\circ}$, Jardim Jalisco,

CEP 27510-096, Resende, RJ, Brazil

E-mail: joaoendocrino@gmail.com

Received: 7 July 2020

Accepted: 11 August 2020

\section{Pneumothorax as a late complication of COVID-19}

\author{
João Guimarães Ferreira ${ }^{(1 *}$, Cristiane Rapparini ${ }^{(1)}{ }^{2 *}$, Bruno Moreno \\ Gomes ${ }^{\left({ }^{1}\right.}$, Luiz Alexandre Cabral Pinto ${ }^{(1}$, Mário Sérgio da Silva e Freire ${ }^{(1)}$
}

\section{ABSTRACT}

In late 2019, a novel coronavirus initially related to a cluster of severe pneumonia cases in China was identified. COVID-19 cases have rapidly spread to multiple countries worldwide. We present a typical laboratory confirmed case of COVID-19 pneumonia, that was hospitalized due to hypoxemia but did not require mechanical ventilation. Although initially the patient was evaluated with a favorable outcome, in the third week of the disease, the symptomatology deteriorated due to a massive hypertensive pneumothorax with no known previous risk factor. Since the first cases of COVID-19 have been described, pneumothorax was characterized as a potential, though uncommon, complication. It has been reported that diffuse alveolar injury caused by SARS-CoV-2 can cause alveolar rupture, produce air leakage and interstitial emphysema. Although uncommon, pneumothorax should be listed as a differential diagnosis for COVID-19 patients with sudden respiratory decompensation. As a life-threatening event, it requires prompt recognition and expeditious treatment.

KEYWORDS: COVID-19. SARS-CoV-2. Pneumonia. Pneumothorax.

\section{INTRODUCTION}

Human coronavirus are important pathogens of respiratory tract infections and are well recognized etiological agents of the common cold. Zoonotic coronaviruses have emerged in recent years to cause human outbreaks, such as the Severe Acute Respiratory Syndrome (SARS) in 2003 and the Middle East Respiratory Syndrome (MERS) in 2012. The SARS-CoV outbreak occurred in 2002-2003, affecting 8,096 patients and spreading from China to 29 countries, with a case-fatality ratio of $9.6 \%{ }^{1}$. MERS-CoV originated in Saudi Arabia in 2012 and was reported in more than 27 countries across the Middle East, Africa, Europe and Asia, with 2,040 confirmed cases and 712 deaths (case-fatality over $30 \%)^{2}$.

In late 2019, a novel coronavirus initially related to a cluster of severe pneumonia cases in Wuhan, China, was identified. The outbreak was declared a Public Health Emergency of International Concern on January $30^{\text {th }}$, 2020 by the World Health Organization. On February $11^{\text {th }}, 2020$, WHO named the new coronavirus disease as COVID-19. The International Committee on Taxonomy of Viruses announced the name of the new virus as Severe Acute Respiratory Syndrome Coronavirus 2 or SARS-CoV-2, because the virus is genetically related to the coronavirus responsible for the SARS outbreak in $2002^{3}$. On March $11^{\text {th }}, 2020$, WHO declared COVID-19 a pandemic, with the increase in the number of cases and countries and territories affected and the sustained risk of further global spread. Since the beginning of the pandemic, the world has registered 18,902,735 confirmed cases with 709,511deaths ${ }^{4}$. In February $25^{\text {th }}, 2020$, the first COVID-19 case was confirmed in Brazil, identified 
as an imported case from Italy ${ }^{5}$. Brazil is currently the epicenter of the pandemic, having totaled 2,859,073 cases with 97,256 deaths on August $7^{\text {th }}, 2020^{4}$.

The clinical presentation of COVID-19 has ranged from mild symptoms, or even asymptomatic cases, to severe illness. Common symptoms consist of fever, cough, fatigue, sore throat, myalgias, shortness of breath, loss of smell and taste and gastrointestinal symptoms, such as diarrhea ${ }^{6,7}$. Patients with a mild presentation of the disease usually progress over a week. Approximately $80 \%$ of laboratory confirmed patients have had mild to moderate disease. On the other hand, patients with more severe disease comprise $14 \%$ of the cases, with progressive tachypnea and dyspnea after five to eight days from the beginning of the symptoms, low blood oxygen saturation, and/or lung infiltrates in $>50 \%$ of the lungs. Six percent of the patients are critical. A large fraction of patients with severe and critical disease required oxygen supplementation, with nasal cannula, Venturi mask, high flow nasal cannula, noninvasive ventilation or even mechanical ventilation. Individuals at higher risk for severe disease and death include people aged over 60 years and those with comorbidities such as hypertension, diabetes, cardiovascular disease, chronic respiratory disease and cancer ${ }^{6,7}$.

Chest imaging is abnormal in a great proportion of patients, even among asymptomatic patients. Chest radiographs exhibit a sensitivity greater than $65 \%$, whereas chest CT sensitivity exceeds $90 \%{ }^{8,9}$. Initially, the hallmarks of COVID-19 infection on imaging exams are bilateral and peripheral ground-glass and opacities. Over time after the onset of symptoms, CT findings are more frequent and include consolidations, bilateral and peripheral disease, greater lung involvement and impairment, linear opacities, "crazy-paving" pattern and the "reverse halo"sign ${ }^{10}$.

Regarding laboratory abnormalities in patients with COVID-2019 infections, the most frequent findings for those who need admission to the intensive care unit are leukocytosis, higher neutrophil count, lymphopenia, increased values of CRP, LDH, aminotransferases, total bilirubin, creatinine, cardiac troponin, procalcitonin and D-dimer. It has also been reported that non-survivors developed more frequently lymphopenia and leukocytosis, along with abnormal high values of D-dimer, blood urea nitrogen and creatinine ${ }^{11}$.

Evidence-based therapy and supportive care in ICU are the mainstay for the management of severe COVID-19. Patients with pneumonia, especially those in severe conditions, may present with coinfections or cross-infections of bacterial pathogens and clinicians should empirically give antibacterial therapy to patients that initially have severe diseases (extensive pneumonia, respiratory failure, hypotension and fever), or present with a clinical deterioration after an initial improvement, or failed to improve after 3 to 5 days. All hospitalized COVID-19 patients should receive thromboprophylaxis, yet a more aggressive individualized strategy might be required in selected cases. Corticosteroids are recommended for severely ill patients on oxygen supplementation or ventilatory support. Observational data suggest that immunomodulation might be helpful to downregulate the inflammatory responses and reduce lung damage ${ }^{12,13}$.

Atypical presentations of COVID-19 disease progression have been described, often misleading the correct approach. Among the uncommon presentations of COVID-19 pneumonia, pneumothorax has rarely been characterized ${ }^{14}$. Some of the patients who develop pneumothorax have risk factors, such as chronic obstructive pulmonary disease (COPD) and are on mechanical ventilation, which can elicit doubt regarding the genesis of this complication. The absence of CT images before the acute event of the pneumothorax can make its pathophysiology difficult to interpret. Herein, we present a typical and laboratory confirmed case of COVID-19 pneumonia, with clinical course deterioration during the third week of the disease due to a massive hypertensive pneumothorax with no known previous risk factor.

\section{METHODS}

The patient provided written informed consent.

A commercial kit (allPLex Seegene-100 T, Seegene Brazil, Belo Horizonte, MG, Brazil) based on the Charité Virology Protocol, Berlin, was employed for the real-time reverse-transcriptase polymerase chain reaction (RT-PCR) for SARS-CoV-2.

A rapid chromatographic immunoassay was used for the qualitative detection of SARS-CoV-2 IgG and IgM antibodies (Biotest, Hangzhou Biotest Biotech Co, Ltd, Hangzhou, China).

Chest CT was performed on a GE Multi-slice Tomograph (16 channels), with fine cuts, high resolution images and multiplanar reformatting, without intravenous contrast infection (Protocol UW-Madison / Madison School of Medicine and Public Health).

\section{CASE REPORT}

A 45-year-old man, measuring $178 \mathrm{~cm}$ in height and weighing $83 \mathrm{~kg}$, sought medical assistance on May $12^{\text {th }}$, 2020 with a history of eight days of burning and redness in his eyes and a $38.2{ }^{\circ} \mathrm{C}$ of body temperature. On the $4^{\text {th }}$ day after the onset of symptoms, he attended a clinic and started levofloxacin, based on a presumptive diagnosis of sinusitis. 
On the $5^{\text {th }}$ day, he presented fatigue and myalgia. On the $7^{\text {th }}$ day, he had sore throat, rhinorrhea and loss of smell. On the same day, he noticed shortness of breath, chest tightness and a mild dry cough. He was admitted to the hospital the next day. His past medical history was unremarkable. He denied any contact with diagnosed COVID-19 patients, or previous tobacco use.

A CT scan obtained on the $8^{\text {th }}$ day revealed multiple pulmonary ground-glass opacities with peripheral and subpleural distribution and septal thickening, predominantly in the posterior segments of the inferior lobes (Figure 1). During his first admission, on the $8^{\text {th }}$ day, his laboratory blood tests showed lymphopenia (leucocytes $-6,940 / \mathrm{mm}^{3}$, neutrophils $-5,691 / \mathrm{mm}^{3}(82 \%)$, lymphocytes $-972 / \mathrm{mm}^{3}$ (14\%), basophiles - 69 (1\%), monocytes - 208 (3\%), C-reactive protein (CRP) - 160,8 mg/L ( $<6 \mathrm{mg} / \mathrm{L})$, D-dimer - $578 \mathrm{ng} / \mathrm{mL}(<500 \mathrm{ng} / \mathrm{mL})$, alanine aminotransferase (ALT) - $38 \mathrm{U} / \mathrm{L}(5-40 \mathrm{U} / \mathrm{L})$, aspartate aminotransferase (AST) $-49 \mathrm{U} / \mathrm{L}$ (8-40 U/L), lactate dehydrogenase (LDH) - $857 \mathrm{UI} / \mathrm{L}$ (240-480 UI/L), creatinine - $1.0 \mathrm{ng} / \mathrm{dL}$. The cardiac troponin I was negative. His arterial blood gases before oxygen was delivered through a nasal cannula showed an oxygen saturation of $91 \%, \mathrm{PaO} 2-56 \mathrm{mmHg}$, $\mathrm{PaCO} 2-27 \mathrm{mmHg}$. A rapid diagnostic test (nasopharyngeal swab) for influenzae A and B was negative. Nasopharyngeal/ oropharyngeal swabs for SARS-CoV-2 analyzed by RT-PCR collected on the $8^{\text {th }}$ day were positive. A rapid chromatographic immunoassay for COVID-19, on the $9^{\text {th }}$ day was positive for IgM and negative for IgG.

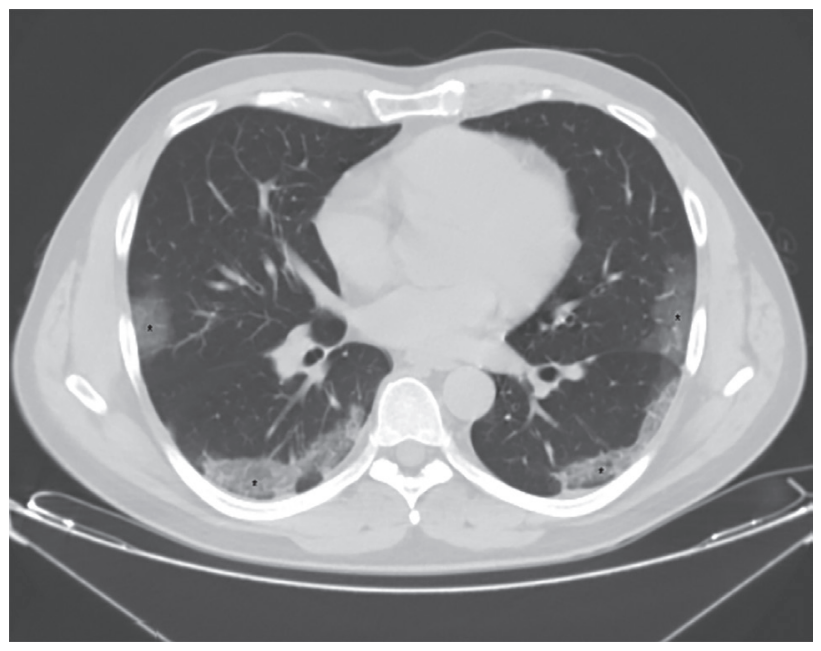

Figure 1 - CT scan showing multiple pulmonary ground-glass opacities with peripheral and subpleural distribution and septal thickening ("crazy-paving" pattern), predominantly in the posterior segments of the inferior lobes, with less than $25 \%$ of lung involvement.

The patient was placed in a general COVID-19 ward and stabilized with oxygen supplementation through a nasal cannula, three to five liters per minute, in dorsal decubitus, evolving with clinical improvement. Methylprednisolone, $250 \mathrm{mg}$ intravenously (IV) in the first day and $125 \mathrm{mg}$ daily in the subsequent days, enoxaparin $80 \mathrm{mg}(1 \mathrm{mg} / \mathrm{kg})$ subcutaneously once a day, azithromycin and ceftriaxone were administered for five days. Central venous access was not required at any time. After five days in the hospital, he was discharged home with oral azithromycin and amoxycillin-clavulanate for five additional days.

The general condition of the patient improved, though he was still felling some shortness of breath on moderate efforts. However, on the $19^{\text {th }}$ day, in the morning, after sneezing, he felt a sudden deep pressure on his thorax, with a sharp pain in the lower part of his left hemithorax, which was aggravated by inspiration.

He was taken to the hospital again. On examination, he had respiratory distress, tachypnea and restlessness. Blood pressure was $130 / 80 \mathrm{mmHg}$, with no jugular vein distension. On auscultation, vesicular murmur was diminished on the left side of the thorax. Arterial blood gases showed an oxygen saturation of $92 \%, \mathrm{PaO} 2-58 \mathrm{mmHg}$ and $\mathrm{PaCO} 2-20 \mathrm{mmHg}$. Laboratory workup showed CRP $9.7 \mathrm{mg} / \mathrm{L}$ and D-dimer - $757 \mathrm{ng} / \mathrm{mL}$.

A chest radiography was immediately obtained and revealed a massive hypertensive pneumothorax of the left lung (Figure 2). A chest drain was successfully inserted promoting the drainage of the left lung, being substituted for another drain because of poor positioning on the third day after its insertion. After a further two days, another

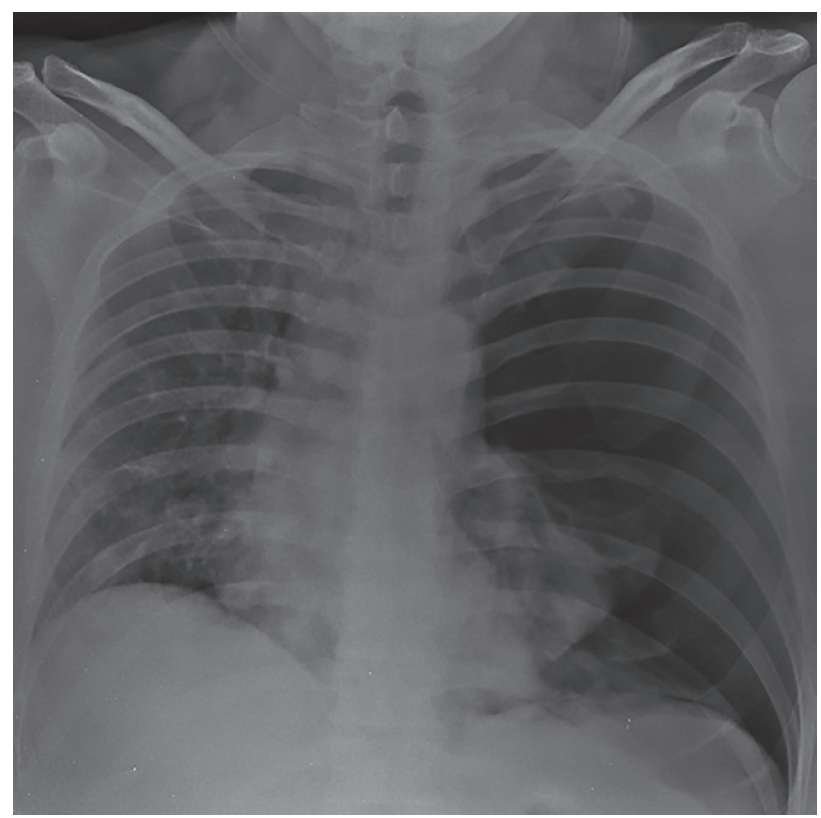

Figure 2 - Massive hypertensive pneumothorax of the left lung, with collapse of the same lung and contralateral deviation of the mediastinal structures. The right lung shows mild alveolar and reticular opacities, notably on the base. 
chest radiography was obtained, which showed reexpansion of the left lung after drainage (Figure 3). The patient was discharged home again.

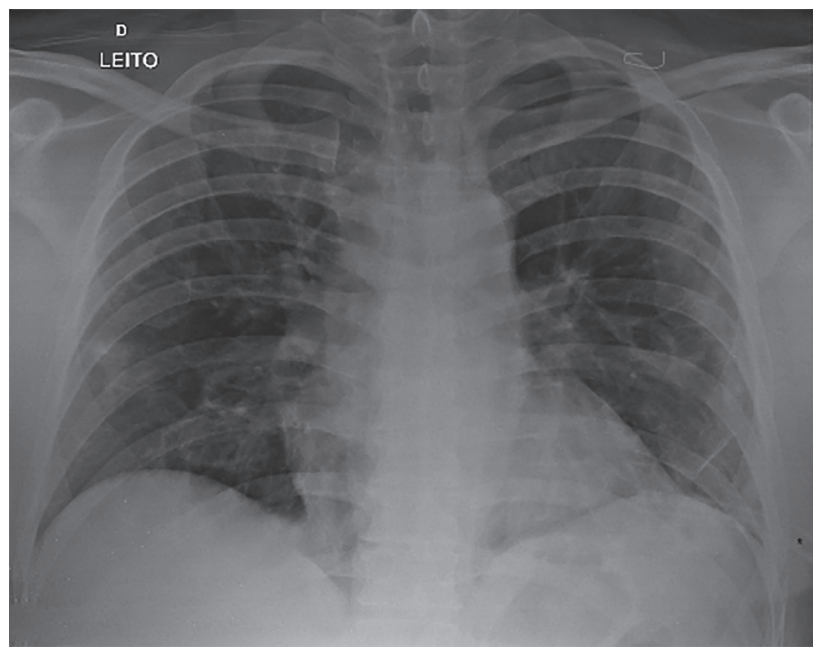

Figure 3 - Reexpansion of the left lung with the insertion of a drain on the left hemithorax.

\section{DISCUSSION}

Pneumothorax consists of the accumulation of air in the pleural space between the parietal pleura and the visceral pleura lining the lung. A primary spontaneous pneumothorax can occur with no precipitating event, while a secondary pneumothorax is a complication of underlying lung disease or trauma. Among lung diseases associated with pneumothorax, emphysema, cystic fibrosis, granulomatous inflammation, necrotizing pneumonia, pulmonary fibrosis, eosinophilic granulomatous disease, sarcoidosis and lung cancer are listed. Patients on mechanical ventilation can develop a pneumothorax due to high local pressure disrupting the lung tissue ${ }^{15}$.

Massive pneumothorax is an acute, life-threatening event which requires prompt diagnosis and immediate chest drainage. A careful physical examination and a high index of suspicion must be employed. Amidst the current COVID-19 pandemic, patients with respiratory failure in emergency rooms are usually subjected to COVID-19 protocols, with the possibility of positive-pressure ventilation, which can aggravate the clinical course of a pneumothorax.

Since the first cases of COVID-19 have been described, pneumothorax was characterized as a potential, though uncommon, complication. Chen et al. described only one patient, out of 99 COVID-19 confirmed cases with pneumothorax ${ }^{16}$. Yang et al., in an autopsy study consisting of 92 patients, found one case with the same diagnosis ${ }^{17}$. Salehi et al. ${ }^{18}$, reviewing CT findings, described pneumothorax as an uncommon finding.
Whereas some patients with COVID-19 developed pneumothorax in association with risk factors, such as mechanical ventilation, in others the only factor was the viral pneumonia itself ${ }^{19}$. In our patient, this is a strong possibility, as he did not present with any previous respiratory diseases or was subjected to positive-pressure ventilation. Moreover, his first chest CT did not display fibrosis, bullae or emphysematous changes which could predispose him to a pneumothorax ${ }^{15}$.

Diffuse alveolar injury caused by SARS-CoV-2 can lead to alveolar rupture, which in turn can produce air leakage and interstitial emphysema. Besides diffuse alveolar damage with cellular fibromyxoid exudates, the presence of multinucleated syncytial cells with atypical enlarged pneumocytes were identified in the inner alveolar spaces, showing viral cytopathic-like changes. Findings typical of the acute respiratory distress syndrome, with desquamation of pneumocytes and hyaline membrane formation, can be present as well ${ }^{20}$.

In conclusion, this paper reports a typical confirmed case of COVID-19 pneumonia, with hypoxemia that did not require mechanical ventilation, with an initial apparent favorable recovery. This case highlights an uncommon clinical scenario of spontaneous pneumothorax as a late complication of COVID-19 pneumonia.

\section{ACKNOWLEDGMENTS}

The authors thank the Hospital Unimed Resende, Resende (RJ), Brazil, and Cristóvão de Albuquerque, who kindly reviewed the final version of the manuscript.

\section{AUTHORS' CONTRIBUTIONS}

Ferreira JG and Rapparini C contributed to study concept and design; Gomes BM contributed to selection and description of imaging data. All authors contributed to drafting of the manuscript.

\section{CONFLICT OF INTERESTS}

All authors have nothing to disclose.

\section{REFERENCES}

1. World Health Organization. Emergencies preparedness, response: summary of probable SARS cases with onset of illness from 1 November 2002 to 31 July 2003. [cited 2020 Aug 11]. Available from: https://www.who.int/csr/sars/country/table2004_04_21/en/

2. Chafekar A, Fielding BC. MERS-CoV: understanding the latest human coronavirus threat. Viruses. 2018;10:93. 
3. Coronaviridae Study Group of the International Committee on Taxonomy of Viruses. The species severe acute respiratory syndrome related coronavirus: classifying 2019-nCoV and naming it SARS-CoV-2. Nature Microbiol. 2020;5:536-44.

4. World Health Organization. Coronavirus disease (COVID-19): situation report - 200. [cited 2020 Aug 11]. Available from: https://www.who.int/docs/default-source/coronaviruse/ situation-reports/20200807-covid-19-sitrep-200. pdf?sfvrsn=2799bc0f_2

5. Cimerman S, Chebabo A, Cunha CA, Rodríguez-Morales AJ. Deep impact of COVID-19 in the healthcare of Latin America: the case of Brazil. Braz J Infect Dis. 2020;24:93-5.

6. Wang D, Hu B, Hu C, Zhu F, Liu X, Zhang J, et al. Clinical characteristics of 138 hospitalized patients with 2019 novel coronavirus-infected pneumonia in Wuhan, China. JAMA. 2020;323:1061-9.

7. Berlin DA, Gulick RM, Martinez FJ. Severe Covid-19. N Engl J Med. 2020 In Press.

8. Wong HY, Lam HY, Fong AH, Leung ST, Chin TW, Lo CS, et al. Frequency and distribution of chest radiographic findings in COVID-19 positive patients. Radiology. 2020;296:E72-8.

9. Fang Y, Zhang H, Xie J, Lin M, Ying L, Pang P, et al. Sensitivity of chest CT for COVID-19: comparison to RT-PCR. Radiology. 2020;296:E115-7.

10. Bernheim A, Mei X, Huang M, Yang Y, Fayad ZA, Zhang N, et al. Chest CT findings in coronavirus disease-19 (COVID-19): relationship to duration of infection. Radiology. 2020;295:68591.
11. Lippi G, Plebani M. Laboratory abnormalities in patients with COVID-2019 infection. Clin Chem Lab Med. 2020;58:1131-4.

12. Horby P, Lim WS, Emberson JR, Mafham M, Bell JL, Linsell L, et al. Dexamethasone in hospitalized patients with Covid-19: preliminary report. N Engl J Med. 2020 In Press.

13. Li L, Li R, Wu Z, Yang X, Zhao M, Liu J, et al. Therapeutic strategies for critically ill patients with COVID-19. Ann Intensive Care. 2020;10:45.

14. Rohailla S, Ahmed N, Gough K. SARS-CoV-2 infection associated with spontaneous pneumothorax. CMAJ 2020;192:E510.

15. McCool FD. Diseases of the diaphragm, chest wall, pleura and mediastinum. In: Goldman L, Schafer AI, editors. GoldmanCecil medicine. $26^{\text {th }}$ ed. Philadelphia: Elsevier; 2020. p.602-12.

16. Chen N, Zhou M, Dong X, Qu J, Gong F, Han Y, et al. Epidemiological and clinical characteristics of 99 cases of 2019 novel coronavirus pneumonia in Wuhan, China: a descriptive study. Lancet. 2020;395:507-13.

17. Yang F, Shi S, Zhu J, Shi J, Dai K, Chen X. Analysis of 92 deceased patients with Covid-19. J Med Virol. 2020 In Press.

18. Salehi S, Abedi A, Balakrishnan S, Gholamrezanezhad A. Coronavirus disease 2019 (COVID-19): a systematic review of imaging findings in 919 patients. AJR Am J Roentgenol. 2020;215:87-93.

19. Ucpinar BA, Sahin C, Yanc U. Spontaneous pneumothorax and subcutaneous emphysema in COVID-19 patient: case report. J Infect Public Health 2020;13:887-9.

20. Xu Z, Shi L, Wang Y, Zhang J, Huang L, Zhang C, et al. Pathological findings of COVID-19 associated with acute respiratory distress syndrome. Lancet Respir Med. 2020;8:420-2. 